\title{
Efeitos de Histórias Experimentais e de Esquemas de Reforço sobre o Seguir Regras ${ }^{1}$
}

\author{
Valdete Leal de Oliveira \\ Luiz Carlos de Albuquerque ${ }^{2}$ \\ Universidade Federal do Pará
}

\begin{abstract}
RESUMO - Investigando variáveis que podem interferir no seguir regras, 16 universitários foram expostos a um procedimento de escolha segundo o modelo. A tarefa: apontar cada um dos três estímulos de comparação, em seqüência. A Fase 1 era de linha de base; na Fase 2, a seqüência correta era estabelecida por contingências; e na Fase 3 era apresentada a regra discrepante das contingências. Manipulou-se os esquemas utilizados para reforçar as seqüências corretas nas Fases 2 e 3. Para os Grupos 1 e 2, os esquemas eram CRF na Fase 2, CRF na Fase 3 do Grupo 1 e FR 3 na Fase 3 do Grupo 2. Para os Grupos 3 e 4, os esquemas eram FR 3 na Fase 2, FR 3 na Fase 3 do Grupo 3 e CRF na Fase 3 do Grupo 4. A história construída na Fase 2 e o esquema na Fase 3 interferiram no seguir regras.
\end{abstract}

Palavras-chave: regras; contingências; histórias experimentais; esquemas de reforço; insensibilidade às contingências programadas.

\section{Effects of Experimental Histories and Reinforcement Schedules on Rule Following}

\begin{abstract}
In an investigation of variables that may interfere with rule-following, 16 college students were exposed to a matching-to-sample procedure, where they had to point, in sequence, to three comparison stimuli. Baseline was referred in Phase 1. In Phase 2, the correct sequence was established by contingencies, and in Phase 3 the discrepant rule to contingencies was presented. The reinforcement schedules in Phases 2 and 3 were manipulated as follows: For Groups 1 and 2, a CRF schedule was used in Phase 2 and also in Phase 3 of Group 1; schedule FR3 was employed in Phase 3 of Group 2. For Groups 3 and 4, the schedules were FR3 in Phase 2, FR3 in Phase 3 of Group 3, and CRF in Phase 3 of Group 4. The history constructed in Phase 2, as well as the schedule in Phase 3, interfered with rule-following.
\end{abstract}

Key words: rules; contingencies; experimental histories; reinforcement schedules; insensibility to programmed contingencies.

Skinner (1969), ao comparar o comportamento controlado por regras com o comportamento controlado por contingências ${ }^{3}$, sugeriu que uma das diferenças entre esses comportamentos é que, quando as contingências mudam, o comportamento estabelecido por regras pode não mudar, no sentido de acompanhar as mudanças nas contingências. Um dos primeiros estudos a testar essa proposição foi o realizado por Shimoff, Catania e Matthews (1981). Nesse estudo, estudantes universitários foram expostos a um es-

1 Este trabalho foi baseado nos dados da Dissertação de Mestrado do primeiro autor apresentada em 2004 no Programa de Pós-Graduação em Teoria e Pesquisa do Comportamento/UFPA e realizada sob orientação do segundo autor. $\mathrm{O}$ trabalho foi realizado com o auxílio financeiro da CAPES, em forma de bolsa concedida ao primeiro autor, e do CNPq.

2 Endereço: Rua Oliveira Belo, 238/1702, Umarizal, Belém, PA, Brasil 66050-380.E-mail: 1calbu@ufpa.br

3 O comportamento é controlado por regras (estímulos antecedentes verbais que descrevem contingências) quando ele é estabelecido por uma regra e ocorre independentemente de suas conseqüências imediatas. E o comportamento é controlado por contingências quando ele é estabelecido por suas consequiências imediatas e ocorre independentemente de uma descrição antecedente das próprias contingências (Albuquerque, 2001). quema de reforço diferencial de baixas taxas - 3s (DRL 3s) superposto a um esquema de intervalo variável - 15s (VI $15 \mathrm{~s})$. Os participantes foram distribuídos em dois grupos. O Grupo de Instrução ${ }^{4}$ era instruído a pressionar um botão lentamente. O Grupo de Modelagem não era exposto a tal instrução. Após 10 minutos de sessão, a contingência DRL era retirada para os dois grupos. Com essa mudança nas contingências, um aumento na taxa de respostas levava a um aumento no número de reforços obtidos. Quando o responder foi estabelecido por instrução, a retirada do esquema DRL levou a um aumento na taxa de respostas em apenas quatro dos 10 participantes. Quando o responder foi estabelecido por modelagem, a retirada do esquema DRL levou a um aumento na taxa de respostas em seis dos sete participantes.

Com base nos resultados desse estudo e em resultados similares encontrados em um estudo anterior (Matthews, Shimoff, Catania \& Sagvolden, 1977), Shimoff e cols. (1981) concluíram que o comportamento estabelecido por regras tem menor probabilidade de mudar acompanhando

4 No presente estudo, os termos "regra" e "instrução" são usados como sinônimos, embora o termo instrução seja usado em situações mais restritas, como nas descrições dos procedimentos e resultados de pesquisas. 
mudanças nas contingências do que o comportamento estabelecido por modelagem e propuseram que a insensibilidade ${ }^{5}$ do comportamento controlado por regras às contingências de reforço programadas seria uma propriedade definidora desse comportamento.

No entanto, de acordo com alguns autores (Cerutti, 1989; Hayes, Zettle \& Rosenfarb, 1989; Newman, Buffington \& Hemmes, 1995), essa insensibilidade não seria uma propriedade definidora do comportamento controlado por regras. Newman e cols. (1995), por exemplo, propuseram que o seguimento de regra seria mantido mais como um resultado do esquema de reforço programado para reforçar o seguimento e o não-seguimento de regra do que uma propriedade inerente às próprias regras. Para avaliar essa possibilidade, distribuíram 18 estudantes universitários em seis grupos experimentais. Inicialmente os participantes foram expostos a uma instrução que descrevia que às vezes a melhor maneira de ganhar fichas era seguindo a instrução; outras vezes era fazendo o oposto ao descrito pela instrução. Uma tentativa era iniciada com a apresentação da instrução especificando que o participante deveria pegar um pino (esquerdo ou direito) e era encerrada após a emissão dessa resposta. As respostas eram reforçadas de acordo com um esquema de reforço contínuo (CRF) nos Grupos 1 e 2, com um esquema de razão fixa - 2 (FR 2) nos Grupos 3 e 4 e com um esquema FR 3 nos Grupos 5 e 6. Cada grupo era exposto a cinco fases. Para os Grupos 1,3 e 5, a instrução correspondia às contingências em 100, 50, 0, 50 e 100\% das tentativas das Fases 1, 2, 3, 4 e 5, respectivamente. Para os Grupos 2, 4 e 6, a instrução correspondia às contingências em $0,50,100,50$ e $0 \%$ das tentativas das Fases 1, 2, 3, 4 e 5, respectivamente.

Nos Grupos 1 e 2, os seis participantes seguiram a instrução nas fases $100 \%$, variaram os seus desempenhos nas fases $50 \%$ e abandonaram o seguimento da instrução nas fases $0 \%$. Nos Grupos 3, 4, 5 e 6, oito participantes seguiram a instrução em mais de $96 \%$ das tentativas das fases e quatro variaram os seus desempenhos (isso é, ora seguiram ora não seguiram a instrução, independentemente das fases). Newman e cols. (1995) concluíram que o seguimento de regras tem maior probabilidade de ser mantido quando o esquema que reforça o seguimento ou o não-seguimento de regra é um esquema de reforço intermitente do que quando é um esquema de reforço contínuo. De acordo com os autores, isso tenderia a ocorrer porque as contingências do esquema de reforço intermitente são mais difíceis de serem discriminadas do que as contingências do esquema de reforço contínuo.

Contudo, entre alguns dos estudos que utilizaram esquema de reforço intermitente, há tanto evidências mostrando que o seguimento de regras pode ser mantido (Albuquerque, de Souza, Matos \& Paracampo, 2003; Albuquerque, Matos, de Souza \& Paracampo, 2004; Kaufman, Baron \& Koop, 1966; Wulfert, Greenway, Farkas, Hayes \& Dougher, 1994), quanto há evidências mostrando que esse comportamento pode deixar de ocorrer (Joyce \& Chase, 1990; LeFrancois, Chase \& Joyce, 1988; Otto, Torgrud \& Holborn, 1999). Do

5 O termo insensibilidade é usado no presente estudo para descrever o comportamento que não é afetado por suas consequiências imediatas, em uma determinada situação particular. mesmo modo, entre alguns dos estudos que utilizaram esquema de reforço contínuo, há tanto evidências mostrando que o seguimento de regras pode ser mantido (Albuquerque, Reis \& Paracampo, 2006; Martinez \& Tomayo, 2005; Monteles, Paracampo \& Albuquerque, 2006; Paracampo, de Souza, Matos \& Albuquerque, 2001), quanto há evidências mostrando que esse comportamento pode deixar de ocorrer (Albuquerque \& Silva, 2006; DeGrandpre \& Buskist, 1991; Paracampo \& Albuquerque, 2004; Santos, Paracampo \& Albuquerque, 2004). Tais evidências sugerem que, além do esquema utilizado para reforçar o seguimento e o não-seguimento de regras, outros fatores podem interferir no seguimento de regras.

Por exemplo, com o objetivo de investigar os efeitos de uma história experimental de reforço intermitente para o seguimento de regra correspondente sobre o seguimento subseqüente de uma regra discrepante das contingências, Albuquerque e cols. (2003) expuseram 16 universitários a um procedimento de escolha de acordo o modelo. Em cada tentativa, um estímulo modelo e três de comparação eram apresentados ao participante, que deveria apontar para os três estímulos de comparação, em sequiência. Cada estímulo de comparação possuía apenas uma dimensão - cor (C), espessura (E) ou forma (F) - em comum com o modelo e diferia nas demais. No Experimento 1, Condição 1, quatro participantes foram expostos à instrução correspondente (especificava a seqüência CEF) e depois à instrução discrepante (especificava FCE). Na Condição 2, outros quatro participantes foram expostos às instruções discrepante - correspondente - discrepante, nessa ordem. Nas duas condições, a sequiência CEF era reforçada (com pontos trocáveis por dinheiro) em FR 4. Diferente do Experimento 1, no Experimento 2 foi demonstrado controle pelas contingências antes da apresentação das instruções. No início das Fases 1, 2, 3 e 4, os oito participantes foram expostos às instruções mínima (não especificava sequiência de respostas), discrepante (especificava FCE), correspondente (especificava EFC) e discrepante, respectivamente. Na Fase 1, a seqüência CEF era estabelecida por reforço diferencial e mantida em FR 4. Nas Fases 2, 3 e 4, a sequiência CEF continuava sendo reforçada em FR 4. Na Fase 3 a seqüência EFC também era reforçada em FR 4, concorrentemente com CEF. A emissão de qualquer outra seqüência não era reforçada.

No Experimento 1, todos os participantes seguiram as instruções. No Experimento 2, a seqüência CEF foi modelada em seis participantes na Fase 1. Desses, quatro seguiram e dois deixaram de seguir as instruções nas fases subseqüentes. Esses dois, ao deixarem de seguir as instruções, passaram a apresentar a sequiência CEF. De acordo com Albuquerque e cols. (2003), os resultados sugerem que o seguimento de regras discrepantes das contingências pode ser mantido mesmo quando esse comportamento não é precedido por uma história experimental de reforço para o seguimento de regra correspondente e mesmo quando se demonstra controle pelas contingências antes da apresentação da regra, isso é, mesmo quando as contingências são fortes.

Considerando os resultados desse estudo, Albuquerque e cols. (2006) procuraram verificar se o seguimento de regras discrepantes das contingências também seria mantido, caso o esquema programado para reforçar o seguimento e o nãoseguimento de regra fosse um esquema de reforço contínuo. 
Para isso, expuseram 16 universitários a um procedimento de escolha de acordo com o modelo que diferiu do usado no estudo de Albuquerque e cols. (2003), porque as sequiências corretas eram reforçadas em CRF e não em FR 4. No Experimento 1 não foi demonstrado controle pelas contingências antes da introdução das instruções, enquanto no Experimento 2 os participantes foram expostos a uma história de reforço contínuo antes de serem expostos às instruções. Todos os oito participantes do Experimento 1 seguiram tanto a instrução correspondente quanto a instrução discrepante das contingências e sete dos oito participantes do Experimento 2 abandonaram o seguimento da instrução discrepante e passaram a responder corretamente, de acordo com suas histórias experimentais e com as contingências em vigor.

De acordo com Albuquerque e cols. (2006), esses resultados, quando comparados com os resultados obtidos no estudo anterior (Albuquerque \& cols., 2003) sugerem que, quando o comportamento de seguir regras discrepantes das contingências de reforço não é precedido por uma história de controle pelas contingências, ele tende a ser mantido, independentemente de o esquema de reforço programado para reforçar o comportamento de seguir ou o de não seguir a regra ser um esquema de reforço contínuo ou um esquema de reforço intermitente. Contudo, quando o comportamento de seguir regra é precedido por uma história de controle pelas contingências de reforço, é mais provável que o comportamento de seguir regras discrepantes seja mantido quando, antes de ser exposto à regra, o participante é exposto a uma história de controle por esquema de reforço intermitente do que quando é exposto a uma história de controle por esquema de reforço contínuo.

Ainda não está claro, no entanto, se o comportamento de seguir regras discrepantes das contingências de reforço depende da história do participante ou do esquema de reforço utilizado para reforçar o não-seguimento de regra, uma vez que nenhum dos estudos comentados até o momento foi planejado com o objetivo de separar os efeitos dessas duas fontes de controle. Diante disso, o presente estudo procurou comparar os efeitos de uma história de reforço intermitente com os efeitos de uma história de reforço contínuo sobre o seguimento subseqüente de regras discrepantes das contingências de reforço e avaliar se a manutenção do seguimento de regras discrepantes das contingências de reforço depende do esquema programado para reforçar o não-seguimento de regra. Isso quando: a) o participante, antes de ser exposto à regra discrepante, é exposto a uma história de CRF, e depois de ser exposto a essa regra, qualquer comportamento é reforçado em CRF, exceto o especificado pela regra [Grupo 1 (CRF/CRF)]; b) o participante, antes de ser exposto à regra discrepante, é exposto a uma história de CRF, e depois de ser exposto a essa regra, qualquer comportamento é reforçado em FR 3, exceto o especificado pela regra [Grupo 2 (CRF/FR 3)]; c) o participante, antes de ser exposto à regra discrepante, é exposto a uma história de reforço em FR 3, e depois de ser exposto a essa regra, qualquer comportamento é reforçado em FR 3, exceto o especificado pela regra [Grupo 3 (FR 3/FR 3)]; e, d) o participante, antes de ser exposto à regra, é exposto a uma história de reforço em FR 3, e depois de ser exposto à regra, qualquer comportamento é reforçado em CRF, exceto o especificado pela regra [Grupo 4 (FR 3/CRF)].
Assim, se o seguimento de regras tem maior probabilidade de ser mantido quando o esquema programado para reforçar o não-seguimento de regra é um esquema de reforço intermitente do que quando é um esquema de reforço contínuo, como sugere a proposição de Newman e cols. (1995), então, deveria ser esperado que o seguimento da regra discrepante fosse mantido nos Grupos 2 (CRF/FR 3) e 3 (FR 3/FR 3), e não se instalasse nos Grupos 1 (CRF/CRF) e 4 (FR 3/CRF). Por outro lado, se o seguimento de regras tem maior probabilidade de ser mantido quando, antes de ser exposto à regra, $\mathrm{o}$ participante é exposto a uma história de reforço intermitente do que quando é exposto a uma história de reforço contínuo, como sugere a proposição de Albuquerque e cols. (2006), então, deveria ser esperado que o seguimento da regra discrepante fosse mantido nos Grupos 3 (FR 3/FR 3) e 4 (FR 3/CRF) e não se instalasse nos Grupos 1 (CRF/CRF) e 2 (CRF/FR 3).

Para tanto, foi utilizado um procedimento de escolha de acordo com o modelo similar ao que tem sido usado por Albuquerque e colaboradores (Albuquerque \& cols., 2003; Albuquerque \& cols., 2006). Esse procedimento foi usado porque permite identificar precisamente, a cada tentativa, se o comportamento emitido está de acordo com a regra ou com as contingências de reforço programadas ou com a história experimental do participante. Desse modo, será dito que o comportamento que se seguir à apresentação da regra está sob o controle da regra, quando esse comportamento for o especificado pela regra e ocorrer independentemente das consequiências programadas no experimento. Será dito que o esquema programado para reforçar o não-seguimento de regra exerceu controle quando o comportamento observado for o reforçado e esse comportamento ocorrer independentemente da regra. E será dito que a história experimental exerceu controle quando o padrão de respostas apresentado em uma dada fase do experimento for apresentado na fase subseqüente, antes mesmo que as conseqüências programadas na nova fase possam exercer algum efeito sobre ele.

\section{Método}

\section{Participantes}

Os participantes foram 16 estudantes universitários, sem experiência prévia nesse tipo de experimento e com idades variando entre 18 e 26 anos. Todos participantes foram voluntários, atendendo a um convite oral do experimentador em diferentes corredores da universidade. $\mathrm{O}$ convite foi feito a alunos do $1^{\circ}$ ao $4^{\circ}$ ano de diversos cursos (exceto o de Psicologia), matriculados em diferentes turmas, procurando-se evitar que pudessem conversar sobre o experimento. Todos os alunos que aceitaram o convite participaram da pesquisa.

\section{Equipamento e material}

Foi utilizada uma mesa de madeira, medindo 150 x 78 x $70 \mathrm{~cm}$. Fixado à mesa, de modo a dividi-la ao meio em todo o seu comprimento, havia um anteparo com espelho unidirecional de $150 \times 60 \mathrm{~cm}$ fixado em uma moldura de madeira e localizado $13 \mathrm{~cm}$ acima do tampo da mesa. No centro do anteparo, junto ao tampo da mesa, havia uma abertura re- 
tangular de 45 x $3 \mathrm{~cm}$. Dois centímetros acima e ao centro dessa abertura havia um contador operado pelo experimentador e com os dígitos voltados para o participante. Visível ao participante estava instalada no anteparo uma lâmpada transparente de 5watts com uma etiqueta de papel com a frase impressa: "Você ganhou um ponto". Uma lâmpada fluorescente de 15 watts estava instalada na borda superior e ao centro do anteparo. Ao lado direito do experimentador, havia duas fitas cassetes, um amplificador e um tape-deck. Conectados ao tape-deck, havia dois fones de ouvido. A mesa estava situada no centro de uma sala.

Os estímulos-modelo e de comparação foram 192 peças de madeira, partes de quatro conjuntos iguais de blocos lógicos (marca FUNBEC), variando em três dimensões: forma (quadrado, círculo, retângulo e triângulo), cor (azul, vermelha e amarela) e espessura (grossa e fina). Essas peças de madeira formavam 40 diferentes arranjos de estímulos, cada um constituído de um estímulo-modelo e três estímulos de comparação. Cada estímulo de comparação apresentava apenas uma dimensão (cor, espessura ou forma) em comum com o estímulo-modelo e diferia nas demais. A combinação dos estímulos era aleatória, assim como a ordem de apresentação dos 40 arranjos. Os estímulos eram apresentados ao participante através da abertura retangular na base do anteparo divisor da mesa, em uma bandeja de madeira em forma de "T". Na parte final do cabo dessa bandeja, rente à base retangular, quatro ripas de madeira formavam um quadrado, no qual era colocado o estímulo-modelo. Na base retangular da bandeja, dividida por ripas de madeira em três quadrados, eram apresentados os três estímulos de comparação. As respostas de escolha emitidas pelos participantes eram registradas pelo experimentador em um protocolo previamente preparado, e também eram gravadas por uma filmadora para análises posteriores. Os reforçadores utilizados eram pontos que eram trocados por dinheiro ao final do experimento. Cada ponto valia $\mathrm{R} \$ 0,05$ (cinco centavos de real).

\section{Procedimento}

Durante as sessões experimentais, participante e experimentador ficavam sentados à mesa de frente um para o outro, separados pelo anteparo divisor da mesa. A lâmpada na borda superior do anteparo ficava constantemente acesa, voltada para o participante, de maneira a assegurar que seu lado apresentasse iluminação em maior intensidade, garantindo que apenas as ações emitidas pelo participante, bem como o arranjo dos estímulos apresentados, pudessem ser observados através do espelho. Os arranjos de estímulos previamente preparados ficavam sobre a mesa, ao lado do experimentador, na ordem em que seriam apresentados em cada tentativa. Para facilitar o manejo dos arranjos, sentado ao lado esquerdo do experimentador, um auxiliar de pesquisa aproximava os arranjos, conforme os mesmos iam sendo apresentados.

Em cada tentativa, o experimentador apresentava um dos 40 arranjos de estímulos. Na presença desses estímulos, o participante deveria apontar para cada um dos três estímulos de comparação, de acordo com o modelo, em uma dada sequiência. Caso a seqüência de respostas emitida estivesse de acordo com as contingências de reforço programadas (seqüência correta), um ponto era acrescentado no contador, a lâmpada transparente era acesa e apagada e a bandeja com o arranjo de estímulos era retirada. Caso a sequiência de respostas fosse incorreta, a lâmpada transparente não era acesa e a bandeja com o arranjo de estímulos era retirada, sem que fosse acrescentado um ponto no contador. Havia um intervalo variável de aproximadamente cinco segundos entre uma tentativa e outra. Os pontos eram registrados cumulativamente, no contador, apenas dentro de uma mesma sessão. No entanto, a partir da segunda sessão, logo após entrar na sala experimental, o participante era informado pelo experimentador sobre o número total de pontos obtidos nas sessões anteriores. Cada sessão, exceto a sessão de linha de base (descrita a seguir), era constituída de 80 tentativas e durava em média 30 minutos. Eram realizadas no máximo duas sessões por dia e o intervalo entre sessões era de aproximadamente 10 minutos. O início e o encerramento de uma sessão eram marcados, respectivamente, pela entrada e saída do participante da sala experimental. O experimentador, em algumas sessões, inicialmente apresentava ao participante uma determinada instrução (descrita a seguir) e em seguida apresentava os arranjos de estímulos, em outras, apresentava apenas os arranjos de estímulos.

\section{Orientações preliminares}

Na primeira sessão, quando participante e experimentador entravam na sala, a bandeja com um arranjo de estímulos estava sobre a mesa, visível ao participante. $\mathrm{O}$ experimentador pedia ao participante para sentar-se na cadeira e, ao lado do participante, sempre apontando com o dedo para cada um dos estímulos a que se referia, dizia:

Este objeto, aqui em cima, é um modelo. Estes três objetos, aqui em baixo, são para você comparar com o modelo. Nós vamos chamar estes três objetos, aqui em baixo, de objetos de comparação. Observe que cada um destes três objetos de comparação tem uma única propriedade comum ao modelo. [Veja: este só tem a espessura comum ao modelo; este aqui só tem a cor comum ao modelo; este aqui só tem a forma igual ao modelo]. Durante a pesquisa você poderá ganhar pontos que serão trocados por dinheiro. Quando você ganhar pontos, os pontos sempre aparecerão aqui neste contador. Veja como os pontos aparecem no contador (o auxiliar, que se encontrava no outro lado da mesa, acionava o contador por cinco vezes). Quando você não ganhar pontos, nenhum ponto será acrescentado no contador. Entendeu?.

Esse procedimento era repetido duas vezes e ocorria apenas no início da primeira sessão. Na segunda vez em que essas orientações preliminares eram apresentadas, o trecho entre colchetes era omitido.

\section{Forma de apresentação das instruções}

A seguir, o experimentador pedia para o participante colocar os fones de ouvido e se deslocava em direção à sua cadeira. Separado do participante pelo anteparo com espe- 
lho unidirecional, o experimentador também colocava os seus fones de ouvido e, dependendo da fase experimental, entregava ao participante, pela abertura na base do anteparo, uma folha de papel contendo uma das seguintes instruções impressas:

Instrução mínima: Instrução que não especificava uma seqüência específica de respostas.

Aponte com o dedo em seqüência para cada um dos três objetos de comparação.

Instrução discrepante: Instrução cujo comportamento de segui-la não era reforçado.

Quando eu mostrar estes objetos para você, você deve fazer o seguinte: Primeiro aponte com o dedo para o objeto de comparação que tem a mesma forma do objeto-modelo. Depois aponte para o objeto de comparação que tem a mesma cor do objetomodelo. Em seguida aponte para o objeto de comparação que tem a mesma espessura do objeto-modelo. Ou seja, você deve apontar primeiro para a mesma forma, depois para a mesma cor e em seguida para a mesma espessura. Entendeu? Repita para mim o que você deve fazer. Fazendo isso, você poderá ganhar pontos que serão mostrados no contador à sua frente. Cada ponto que você ganhar será trocado por $R \$ 0,05$ (cinco centavos de real), mas apenas no final da pesquisa.

Imediatamente após entregar ao participante a folha de papel contendo uma instrução impressa, o experimentador ligava o tape-deck e, por intermédio dos fones de ouvido, o participante passava a ouvir uma fita, previamente gravada, com a voz do experimentador lendo a instrução impressa. $\mathrm{Na}$ gravação, o experimentador solicitava ao participante, ora que acompanhasse a sua leitura, ora que ele lesse sozinho, silenciosamente. Esse procedimento foi usado para garantir que a instrução impressa fosse lida. Desse modo, o participante lia a instrução por três vezes seguidas no início das Fases 1 e 3 e, quando na Fase 3 a segunda ou a terceira sessão teve de ser realizada, lia a instrução uma única vez no início dessas sessões. Após a última leitura, a gravação solicitava ao participante que devolvesse a folha com a instrução. Logo após receber a folha com a instrução, o experimentador removia a bandeja, voltava a apresentar a bandeja com um novo arranjo de estímulos, e dizia: "Comece a apontar".

\section{Delineamento experimental}

Os participantes foram distribuídos em quatro grupos, cada um com quatro participantes. Nos quatro grupos, cada participante era exposto a três fases, como indicado na Tabela 1. A Fase 1 era iniciada com a apresentação da instrução mínima, a Fase 2 com a apresentação de um arranjo de estímulos, uma vez que nessa fase não eram apresentadas instruções, e a Fase 3 com a instrução discrepante das contingências de reforço programadas. Os quatro grupos diferiam quanto ao esquema de reforço utilizado nas Fases 2 e 3. Para o Grupo 1 (CRF/CRF), o esquema era CRF nas Fases 2 e 3; para o Grupo 2 (CRF/FR 3), os esquemas eram CRF na Fase 2 e FR 3 na Fase 3; para o Grupo 3 (FR 3/FR 3), o esquema era FR 3 nas Fases 2 e 3; e para o Grupo 4 (FR 3/CRF), os esquemas eram FR 3 na Fase 2 e CRF na Fase 3. Desse modo, as comparações entre fases permitiam avaliar, em um mesmo participante, o controle pela história experimental, o controle pela regra discrepante e o controle pelo esquema programado para reforçar o não-seguimento da regra. Já as comparações entre grupos permitiam tanto comparar os efeitos da história de reforço contínuo com os efeitos da história de reforço intermitente quanto comparar os efeitos dos esquemas de reforço, sobre o seguimento da regra discrepante. Segue a descrição das fases a que cada participante de cada grupo foi exposto.

Tabela 1. Esquema do procedimento.

\begin{tabular}{|c|c|c|c|c|}
\hline Fases & Grupo 1 (CRF/CRF) & Grupo 2 (CRF/FR 3) & Grupo 3 (FR 3/FR 3) & Grupo 4 (FR 3/CRF) \\
\hline Fase 1 & \multicolumn{4}{|c|}{ Instrução mínima } \\
\hline Seqüência instruída & \multicolumn{4}{|c|}{ Nenhuma } \\
\hline Sequiência reforçada & \multicolumn{4}{|c|}{ Nenhuma } \\
\hline Esquema de reforço & \multicolumn{4}{|c|}{ Nenhum } \\
\hline Fase 2 & \multicolumn{4}{|c|}{ Sem instruções } \\
\hline Seqüência instruída & \multicolumn{4}{|c|}{ Nenhuma } \\
\hline Seqüência reforçada & \multicolumn{4}{|c|}{ CEF ou ECF } \\
\hline Esquema de reforço & \multicolumn{2}{|c|}{ Reforço contínuo } & \multicolumn{2}{|c|}{ Razão fixa 3} \\
\hline Fase 3 & \multicolumn{4}{|c|}{ Instrução discrepante } \\
\hline Seqüência instruída & \multicolumn{4}{|c|}{ FCE } \\
\hline Seqüência reforçada & \multicolumn{4}{|c|}{ CEF, CFE, EFC, ECF e FEC } \\
\hline Esquema de reforço & Reforço contínuo & \multicolumn{2}{|c|}{ Razão fixa 3} & Reforço contínuo \\
\hline
\end{tabular}

Nota: Cada um dos quatro grupos foi formado por quatro participantes. C=resposta à dimensão cor; $E=$ resposta à dimensão espessura; $F=$ resposta à dimensão forma; 


\section{Grupol (CRF/CRF)}

A Fase 1 era constituída de uma única sessão de 10 tentativas de linha de base em relação à qual eram avaliados os efeitos da introdução das consequiências programadas na Fase 2. Portanto, durante a Fase 1, nenhuma resposta era reforçada.

A Fase 2 era constituída de três passos: estabelecimento, extinção e recuperação da seqüência de repostas correta. Nessa fase, apenas uma de duas seqüências de respostas era reforçada e, portanto, considerada correta: a seqüência cor - (C) - espessura - (E) - forma - (F) ou a sequiência ECF. Qual dessas duas seqüências seria reforçada, dependia do desempenho do participante na Fase 1. Se na Fase 1 a seqüência CEF fosse emitida em mais de $50 \%$ das tentativas, a seqüência correta na Fase 2 seria a seqüência ECF e não a seqüência CEF. Caso contrário, a sequiência correta na Fase 2 seria a seqüência $C E F$ e não a seqüência ECF. A seqüência correta na Fase 2 era inicialmente reforçada em CRF. Imediatamente após o participante receber 30 pontos em CRF, a seqüência correta deixava de ser reforçada (extinção) durante 80 tentativas. Depois disso, a seqüência correta voltava a ser reforçada em CRF até a obtenção de mais 120 pontos, quando essa fase era encerrada e o participante era exposto à Fase 3. Essa fase também podia ser encerrada caso o participante passasse 160 tentativas (duas sessões) sem ganhar pontos no passo de estabelecimento ou no passo de recuperação da seqüência correta. Portanto, nesse caso, o participante não era exposto à Fase 3.

Na Fase 3 era reforçada a emissão de qualquer seqüência de três diferentes respostas, exceto a seqüência FCE, especificada pela regra discrepante. Durante a Fase 3, portanto, podiam ser reforçadas as emissões das seguintes seqüências de respostas: CEF, CFE, EFC, ECF e FEC. Essas seqüências eram reforçadas em CRF. A Fase 3 era encerrada quando um dos seguintes critérios fosse atingido, o que ocorresse primeiro: 1) após três sessões serem completadas, isso é, após a ocorrência de 240 tentativas ou 2) após a obtenção de 80 pontos. A participação do estudante no experimento era encerrada quando um desses critérios fosse atingido ou caso não fosse atingido o critério de desempenho para o encerramento da Fase 2.

Grupo 2 (CRF/FR 3)

O Grupo 2 (CRF/FR 3) diferia do Grupo 1 (CRF/CRF) apenas no seguinte aspecto da Fase 3: Na Fase 3 do Grupo 2 (CRF/FR 3), as seqüências CEF, CFE, EFC, ECF e FEC eram reforçadas, desde o início dessa fase, em FR 3. Nesse esquema de razão fixa, cada três emissões consecutivas de uma mesma seqüência correta produzia um ponto no contador. Erros ou a não-emissão consecutiva de uma mesma seqüência correta reiniciavam a razão fixa 3 para obtenção de um ponto.

Grupo 3 (FR 3/FR 3)

O Grupo 3 (FR 3/FR 3) diferia do Grupo 1 (CRF/CRF) apenas nos seguintes aspectos das Fases 2 e 3: Na Fase 2 do Grupo 3 (FR 3/FR 3), a seqüência correta (CEF ou ECF) era inicialmente reforçada em CRF. Imediatamente após o participante receber 10 pontos em CRF, a seqüência correta passava a ser reforçada em FR 2. Depois que o participante tivesse obtido 10 pontos em FR 2, a sequiência correta passava a ser reforçada em FR 3 até a obtenção de 20 pontos em FR 3 (modelagem). Após esse critério ser atingido, a sequiência correta deixava de ser reforçada (extinção) durante 80 tentativas. Em seguida, a seqüência correta voltava a ser reforçada em FR 3 até a obtenção de mais 120 pontos, quando essa fase era encerrada. Na Fase 3 do Grupo 3 (FR 3/FR 3), as seqüências CEF, CFE, EFC, ECF e FEC eram reforçadas, desde o início dessa fase, em FR 3.

\section{Grupo $4(F R 3 / C R F)$}

O Grupo 4 (FR 3/CRF) diferia do Grupo 3 (FR 3/FR 3) apenas porque as seqüências CEF, CFE, EFC, ECF e FEC eram reforçadas em CRF na Fase 3.

\section{Comparação dos registros}

A cada duas sessões, um observador independente comparava o registro feito pelo experimentador com o registro feito pela filmadora. Caso houvesse $100 \%$ de concordância entre os registros, o participante continuaria participando do experimento. Caso contrário, o participante seria descartado. No presente estudo, nenhum participante foi descartado.

\section{Resultados}

Inicialmente serão apresentados os dados dentro de cada fase e, em seguida, serão feitas as comparações entre fases e entre grupos. Todos os 16 participantes apresentaram um desempenho variável na Fase 1. Portanto, como nenhum participante chegou a emitir a seqüência CEF em mais de

Tabela 2. Porcentagens de seqüências de respostas emitidas durante os passos de estabelecimento e recuperação da Fase 2.

\begin{tabular}{|c|c|c|c|c|c|c|c|}
\hline \multirow[t]{2}{*}{ Grupos } & \multirow[t]{2}{*}{ Participantes } & \multicolumn{6}{|c|}{ Seqüências } \\
\hline & & $\mathrm{CEF}$ & CFE & FCE & FEC & $\mathrm{EFC}$ & $\mathrm{ECF}$ \\
\hline 1 & $\mathrm{P} 11$ & 65 & 13 & 14 & 7 & 0,5 & 0,5 \\
\hline \multirow[t]{3}{*}{ (CRF/CRF) } & $\mathrm{P} 12$ & 83 & 1 & 0 & 0 & 0 & 16 \\
\hline & $\mathrm{P} 13$ & 80 & 1 & 3 & 3 & 4 & 9 \\
\hline & $\mathrm{P} 14$ & 72 & 27 & 1 & 0 & 0 & 0 \\
\hline 2 & $\mathrm{P} 21$ & 78 & 5 & 7 & 5 & 2 & 3 \\
\hline \multirow[t]{3}{*}{ (CRF/FR3) } & $\mathrm{P} 22$ & 91 & 2 & 7 & 0 & 0 & 0 \\
\hline & $\mathrm{P} 23$ & 94 & 1 & 1 & 3 & 1 & 0 \\
\hline & $\mathrm{P} 24$ & 80 & 5 & 3 & 1 & 8 & 3 \\
\hline 3 & P31 & 99 & 0 & 0 & 0,5 & 0 & 0,5 \\
\hline \multirow[t]{3}{*}{ (FR3/FR3) } & P32 & 93 & 2 & 1 & 1 & 1 & 2 \\
\hline & P33 & 99 & 0,5 & 0 & 0 & 0 & 0,5 \\
\hline & P34 & 86 & 5 & 2 & 1 & 2 & 4 \\
\hline 4 & $\mathrm{P} 41$ & 73 & 15 & 6 & 3 & 1 & 2 \\
\hline \multirow[t]{3}{*}{ (FR3/CRF) } & $\mathrm{P} 42$ & 72 & 6 & 7 & 7 & 3 & 5 \\
\hline & $\mathrm{P} 43$ & 0 & 100 & 0 & 0 & 0 & 0 \\
\hline & P44 & 76 & 5 & 3 & 4 & 5 & 7 \\
\hline
\end{tabular}

Nota: $\mathrm{C}=$ resposta à dimensão cor; $\mathrm{E}=$ resposta à dimensão espessura; $\mathrm{F}=$ resposta à dimensão forma. 
$50 \%$ das tentativas da Fase 1, a Fase 2 dos quatro grupos foi iniciada reforçando-se diferencialmente a seqüência CEF.

A Tabela 2 mostra a porcentagem de seqüências de respostas emitidas pelos participantes durante os passos de estabelecimento e recuperação da Fase 2. Pode-se observar que, exceto $\mathrm{P} 43$, todos os demais 15 participantes emitiram a sequiência correta $(\mathrm{CEF})$ em mais de $65 \%$ das tentativas desses passos, ou seja, atingiram o critério de desempenho para o encerramento da Fase 2 e, assim, foram expostos à Fase 3. O Participante P43 teve sua participação no experimento encerrada no passo de estabelecimento da seqüência correta da Fase 2, porque permaneceu 160 tentativas sem ganhar pontos durante esse passo.

A Tabela 3 mostra a porcentagem de sequiências de respostas emitidas durante as 80 tentativas do passo de extinção da Fase 2. Observa-se que o Participante P33 foi o único que emitiu a seqüência CEF em $100 \%$ das tentativas da extinção, os demais 14 participantes chegaram a variar os seus desempenhos durante esse passo. Oito participantes [P12, P13 e P14 do Grupo 1 (CRF/CRF), P21, P22, P23 e P24 do Grupo 2 (CRF/FR 3) e P41 do Grupo 4 (FR 3/CRF)] emitiram a seqüência $\mathrm{CEF}$ em menos de $22 \%$ das tentativas desse passo e sete participantes (P11 do Grupo 1 [CRF/CRF), P31, P32, P33 e P34 do Grupo 3 (FR 3/FR 3) e P42 e P44 do Grupo 4 (FR 3/CRF)] fizeram o mesmo em mais de $47 \%$ das tentativas desse passo.

Tabela 3. Porcentagens de seqüências de respostas emitidas durante o passo de extinção da Fase 2.

\begin{tabular}{cccccccc}
\hline Grupos & Participantes & \multicolumn{7}{c}{ Seqüências } \\
\hline 1 & & CEF & CFE & FCE & FEC & EFC & ECF \\
\hline (CRF/CRF) & P11 & 51 & 6 & 21 & 15 & 3 & 4 \\
& P12 & 21 & 18 & 14 & 12 & 19 & 16 \\
& P13 & 21 & 13 & 15 & 16 & 20 & 15 \\
& P14 & 16 & 15 & 19 & 14 & 16 & 20 \\
\hline 2 & P21 & 16 & 16 & 19 & 21 & 15 & 13 \\
(CRF/FR3) & P22 & 21 & 16 & 19 & 12 & 14 & 18 \\
& P23 & 15 & 20 & 15 & 16 & 17 & 17 \\
& P24 & 11 & 17 & 25 & 14 & 19 & 14 \\
\hline 3 & P31 & 85 & 5 & 2 & 3 & 1 & 4 \\
(FR3/FR3) & P32 & 65 & 2 & 10 & 3 & 9 & 11 \\
& P33 & 100 & 0 & 0 & 0 & 0 & 0 \\
& P34 & 47 & 30 & 10 & 1 & 6 & 6 \\
\hline 4 & P41 & 22 & 22 & 22 & 21 & 7 & 6 \\
(FR3/CRF) & P42 & 52 & 26 & 4 & 1 & 11 & 6 \\
& P44 & 94 & 1 & 3 & 2 & 0 & 0 \\
\hline
\end{tabular}

A Tabela 4 mostra os dados da Fase 3, relativos ao número ordinal da tentativa a partir da qual o comportamento de seguir a regra discrepante deixou de ocorrer definitivamente nessa fase. Todos os 15 participantes iniciaram a Fase 3 seguindo a regra. Depois, três continuaram seguindo a regra durante toda a fase [P31 e P33 do Grupo 3 (FR 3/FR 3) e P41 do Grupo 4 (FR 3/CRF)], quatro seguiram a regra durante a maior parte da fase [P21, P22, P23 e P24 do Grupo 2 (CRF/FR $3)]$ e oito deixaram de seguir a regra definitivamente [P11,
P12, P13 e P14 do Grupo 1 (CRF/CRF), P32 e P34 do Grupo 3 (FR 3/FR 3), e P42 e P44 do Grupo 4 (FR 3/CRF)]. Os participantes que deixaram de seguir a regra definitivamente, fizeram isso logo no início dessa fase, isso é, entre a quarta e a décima tentativa.

Tabela 4. Resumo dos principais dados da Fase 3.

\begin{tabular}{ccc}
\hline Grupos & Participantes & $\begin{array}{c}\text { Número ordinal da tentativa a } \\
\text { partir da qual o seguir a regra } \\
\text { deixou de ocorrer definitivamente }\end{array}$ \\
\hline 1 & $\mathrm{P} 11$ & $6^{\mathrm{a}}$ \\
$(\mathrm{CRF} / \mathrm{CRF})$ & $\mathrm{P} 12$ & $4^{\mathrm{a}}$ \\
& $\mathrm{P} 13$ & $5^{\mathrm{a}}$ \\
& $\mathrm{P} 14$ & $10^{\mathrm{a}}$ \\
\hline 2 & $\mathrm{P} 21$ & Não \\
$(\mathrm{CRF} / \mathrm{FR} 3)$ & $\mathrm{P} 22$ & Não \\
& $\mathrm{P} 23$ & Não \\
& $\mathrm{P} 24$ & Não \\
\hline 3 & $\mathrm{P} 31$ & Não \\
$(\mathrm{FR} 3 / \mathrm{FR} 3)$ & $\mathrm{P} 32$ & $5^{\mathrm{a}}$ \\
& $\mathrm{P} 33$ & Não \\
& $\mathrm{P} 34$ & $10^{\mathrm{a}}$ \\
\hline 4 & $\mathrm{P} 41$ & Não \\
(FR3/CRF) & $\mathrm{P} 42$ & $5^{\mathrm{a}}$ \\
& $\mathrm{P} 44$ & $9^{\mathrm{a}}$ \\
\hline
\end{tabular}

Nota: Todos os 15 participantes seguiram a regra discrepante na primeira tentativa da Fase 3. "Não", indica que o seguir a regra não deixou de ocorrer definitivamente, isso é, indica que ele foi mantido durante toda a fase (caso de P31, P33 e P41) ou durante a maior parte da fase (caso de P21, P22, $\mathrm{P} 23$ e P24).

A Tabela 5 mostra as porcentagens de seqüências de respostas emitidas durante a Fase 3 pelos participantes que foram expostos a essa fase. No Grupo 1 (CRF/CRF), observa-se que, após deixarem de seguir a regra na Fase 3, três (P12, P13 e P14) dos quatro participantes passaram a emitir a seqüência correta CEF, modelada na Fase 2, em mais de $89 \%$ das tentativas da Fase 3. O Participante P11, após deixar de seguir a regra, emitiu a sequiência CFE, também correta, em $93 \%$ das tentativas. No Grupo 2 (CRF/FR 3), observa-se que $\mathrm{P} 21, \mathrm{P} 22, \mathrm{P} 23$ e $\mathrm{P} 24$ seguiram a regra, isso é, emitiram a sequiência FCE em 85, 61, 89 e 95\% das tentativas, respectivamente. No Grupo 3 (FR 3/FR 3), observa-se que P31 e P33 seguiram a regra, isso é, emitiram a seqüência FCE em $100 \%$ das tentativas. O Participante P32, após deixar de seguir a regra, emitiu a sequiência correta $\mathrm{CFE}$ em $76 \%$ das tentativas. Já o Participante P34, após deixar de seguir a regra, emitiu a seqüência correta CEF em $96 \%$ das tentativas. Em outras palavras, ao deixar de seguir a regra na Fase 3, emitiu a seqüência modelada na Fase 2 (CEF) por três vezes consecutivas (completando desse modo os requisitos do esquema FR 3), obteve ponto e continuou emitindo essa sequiência. Por último, no Grupo 4 (FR 3/CRF), pôde-se observar que P41 seguiu a regra discrepante das contingências em 100\% das tentativas. Já os Participantes P42 e P44 deixaram de 
seguir a regra discrepante. Ao deixarem de seguir a regra, P42 passou a emitir a seqüência CEF (modelada na Fase 2) em $77 \%$ das tentativas, ao passo que $\mathrm{P} 44$ passou a emitir a seqüência ECF em $87 \%$ das tentativas.

Tabela 5. Porcentagens de sequiências de respostas emitidas durante a Fase 3.

\begin{tabular}{|c|c|c|c|c|c|c|c|}
\hline \multirow[t]{2}{*}{ Grupos } & \multirow[t]{2}{*}{ Participantes } & \multicolumn{6}{|c|}{ Seqüiências } \\
\hline & & CEF & CFE & FCE & FEC & EFC & $\mathrm{ECF}$ \\
\hline 1 & P11 & 1 & 93 & 6 & 0 & 0 & 0 \\
\hline \multirow[t]{3}{*}{ (CRF/CRF) } & $\mathrm{P} 12$ & 95 & 0 & 5 & 0 & 0 & 0 \\
\hline & P13 & 95 & 0 & 5 & 0 & 0 & 0 \\
\hline & P14 & 89 & 1 & 10 & 0 & 0 & 0 \\
\hline 2 & $\mathrm{P} 21$ & 1 & 1 & 85 & 5 & 3 & 5 \\
\hline \multirow[t]{3}{*}{ (CRF/FR3) } & $\mathrm{P} 22$ & 10 & 8 & 61 & 13 & 3 & 5 \\
\hline & $\mathrm{P} 23$ & 2 & 2 & 89 & 4 & 2 & 1 \\
\hline & $\mathrm{P} 24$ & 2 & 0 & 95 & 1 & 1 & 1 \\
\hline 3 & $\mathrm{P} 31$ & 0 & 0 & 100 & 0 & 0 & 0 \\
\hline \multirow[t]{3}{*}{ (FR3/FR3) } & P32 & 1 & 76 & 22 & 1 & 0 & 0 \\
\hline & P33 & 0 & 0 & 100 & 0 & 0 & 0 \\
\hline & P34 & 96 & 0 & 4 & 0 & 0 & 0 \\
\hline 4 & P41 & 0 & 0 & 100 & 0 & 0 & 0 \\
\hline \multirow[t]{2}{*}{ (FR3/CRF) } & $\mathrm{P} 42$ & 77 & 0 & 22 & 1 & 0 & 0 \\
\hline & P44 & 0 & 0 & 12 & 0 & 1 & 87 \\
\hline
\end{tabular}

Nota: $\mathrm{C}=$ Resposta à dimensão cor; $\mathrm{E}=$ resposta à dimensão espessura; $\mathrm{F}=$ resposta à dimensão forma.

Em síntese, os resultados desse experimento mostraram que 15 dos 16 participantes atingiram o critério de desempenho para o encerramento da Fase 2. Desses 15, sete (P21, P22, P23, P24, P31, P33 e P41) seguiram a regra discrepante na Fase 3 e oito deixaram de seguir essa regra (P11, P12, P13, P14, P32, P34, P42 e P44). Dos sete participantes que seguiram a regra discrepante, seis $(\mathrm{P} 21, \mathrm{P} 22, \mathrm{P} 23, \mathrm{P} 24$, P31 e P33) eram dos Grupos 2 (CRF/FR 3) e 3 (FR 3/FR 3 ), para os quais foi programado um esquema de reforço intermitente na Fase 3. Dos oito participantes que deixaram de seguir a regra discrepante, seis $(\mathrm{P} 11, \mathrm{P} 12, \mathrm{P} 13, \mathrm{P} 14, \mathrm{P} 42$ e P44) eram dos Grupos 1 (CRF/CRF) e 4 (FR 3/CRF), para os quais foi programado um esquema de reforço contínuo na Fase 3. Dos sete participantes que seguiram a regra discrepante, três (P31, P33 e P41) seguiram essa regra em 100\% das tentativas e quatro (P21, P22, P23 e P24) fizeram isso entre 61 e $95 \%$ das tentativas. Os três que seguiram a regra discrepante em $100 \%$ das tentativas haviam sido expostos a uma história de reforço intermitente da seqüência CEF na Fase 2, e os quatro que seguiram essa regra em menos de $100 \%$ das tentativas haviam sido expostos a uma história de reforço contínuo da seqüência CEF na Fase 2. Dos oito participantes que deixaram de seguir a regra discrepante na Fase 3, cinco (P12, P13, P14, P34 e P42), após deixarem de seguir essa regra na Fase 3, passaram a emitir a sequiência CEF, modelada na Fase 2.

\section{Discussão}

Os resultados do presente estudo mostraram que, quando o esquema programado para reforçar o não-seguimento da regra era um esquema de reforço intermitente [Grupos 2 (CRF/FR3) e 3 (FR3/FR3)], seis (P21, P22, P23, P24, P31 e P33) dos oito participantes seguiram a regra discrepante; e quando o esquema programado para reforçar o não-seguimento da regra era um esquema de reforço contínuo [Grupos 1 (CRF/CRF) e 4 (FR3/CRF)], seis (P11, P12, P13, P14, $\mathrm{P} 42$ e P44) dos sete participantes deixaram de seguir a regra discrepante. Esses resultados globais poderiam indicar que a manutenção do seguimento de regras discrepantes das contingências depende do esquema programado para reforçar o não-seguimento de regra, como sugere a proposição de Newman e cols. (1995).

Contudo, uma análise mais minuciosa dos resultados pode indicar que outras variáveis, além do esquema programado para reforçar o não-seguimento de regra, também poderiam estar controlando os desempenhos dos participantes no presente estudo. Os resultados dos Participantes P11, P12, P13 e P14 [Grupo 1 (CRF/CRF)] sugerem isso. Três (P12, P13 e P14) desses quatro participantes, após deixarem de seguir a regra na Fase 3, não variaram os seus desempenhos. Ao invés disso, passaram imediatamente a emitir a sequiência $\mathrm{CEF}$, que havia sido modelada na Fase 2. Esses resultados indicam que o desempenho desses três participantes durante a Fase 3 também estava sob controle de suas histórias experimentais. Isso considerando que, embora durante a Fase 3 pudessem ser reforçadas as emissões de cinco seqüências de respostas (CEF, CFE, EFC, ECF e FEC), esses três participantes emitiram exatamente a única (CEF) que havia sido previamente reforçada na Fase 2. O Participante P11, imediatamente após deixar de seguir a regra discrepante, também emitiu a seqüência CEF nas duas tentativas subseqüentes, mas depois passou a emitir a sequiência CFE, que era formalmente semelhante à sequiência $\mathrm{CEF}$ e também era reforçada.

Esse efeito da história experimental é semelhante ao que tem sido chamado de ressurgência de um comportamento previamente reforçado (Dixon \& Hayes, 1998; Epstein \& Skinner, 1980; Lieving, Hagopian, Long \& O'Connor, 2004; Lieving \& Lattal, 2003). Mas o fenômeno observado no início da Fase 3 do Grupo 1 (CRF/CRF) do presente estudo é mais adequadamente descrito como substituição do comportamento especificado pela regra pelo comportamento previamente reforçado (Albuquerque \& cols., 2003). Além disso, diferente do procedimento usado nos estudos que têm investigado o fenômeno de ressurgência ${ }^{6}$ (ver Lieving \& Lattal, 2003), no presente estudo o comportamento que "ressurgiu" na Fase 3 (isso é, o comportamento que substituiu o especificado pela regra) continuava sendo reforçado nessa

6 No procedimento utilizado para investigar esse fenômeno, de modo geral, um comportamento é estabelecido em uma primeira fase. Depois, em uma segunda fase, esse comportamento é colocado em extinção, enquanto um comportamento alternativo é reforçado. Por último, em uma terceira fase, tanto o comportamento estabelecido na primeira fase quanto o comportamento alternativo estabelecido na segunda fase são colocados em extinção. Denomina-se de ressurgência a emissão, na terceira fase (extinção), do comportamento estabelecido na primeira fase. 
fase e esse fato, provavelmente, contribuiu para determinar a sua manutenção.

Essa análise sugere, então, que o seguimento da regra discrepante deixou de ocorrer ao entrar em contato com as consequiências que contradiziam a regra (a não obtenção de pontos, no caso do presente estudo), como tem sido proposto (Galízio, 1979). Mas o comportamento que substituiu o comportamento especificado pela regra, quando este não ocorreu, não foi determinado exclusivamente por suas consequiências. Esse comportamento foi determinado pela história experimental dos participantes (Albuquerque \& cols., 2003). Isso não implica que o controle por essa história, por si só, tenha impedido o controle pela regra discrepante. Depois de emitido, o não-seguimento da regra passou a ser mantido, provavelmente, por suas conseqüências reforçadoras (a obtenção de pontos, no caso do presente estudo). Isso fica mais claro, no entanto, quando os resultados dos participantes do Grupo 1 (CRF/CRF) são comparados com os resultados dos participantes do Grupo 2 (CRF/FR 3).

Os Participantes P21, P22, P23 e P24 [Grupo 2 (CRF/FR 3)] variaram um pouco seus desempenhos no início da Fase 3 , quando o seguimento da regra não foi reforçado. Mas voltaram a seguir a regra, possivelmente porque o não-seguimento da regra não foi reforçado. Mas por que o comportamento de não seguir a regra não foi reforçado? Isso possivelmente ocorreu porque, no caso desses participantes, o esquema programado para reforçar o não-seguimento da regra era de razão fixa (exigia que uma sequiência correta fosse emitida por três vezes consecutivas e a não emissão consecutiva de uma mesma sequiência correta, reiniciavam a razão fixa para obtenção de um ponto) e eles não tinham uma história experimental de seus desempenhos serem reforçados nesse esquema.

Os dados dos Participantes P32 e P34 [Grupo 3 (FR 3/FR 3)] apóiam essa sugestão. Esses participantes tinham uma história experimental de seus desempenhos serem reforçados em FR 3 e essa história, possivelmente, contribuiu para que eles tivessem emitido uma sequiência correta por três vezes consecutivas na Fase 3, quando o seguimento da regra deixou de ocorrer. Ao exercer essa função de determinar a ocorrência do comportamento substituto do comportamento especificado pela regra, essa história possibilitou que as consequiências programadas para reforçar o não-seguimento da regra também pudessem exercer sua função de contribuir para manter a ocorrência desse comportamento.

Mas por que, diferente dos Participantes P32 e P34, os outros dois participantes (P31 e P33) do Grupo 3 (FR 3/FR 3) seguiram a regra? Essa variabilidade também tem sido encontrada em outros estudos que investigaram os efeitos de histórias de controle por esquemas de reforço intermitente sobre o seguimento de regras discrepantes (Albuquerque \& cols., 2003; Albuquerque \& cols., 2004) e não está claro por que ela ocorreu. Os participantes do Grupo 3 (FR 3/FR 3) tinham uma história de exposição a contingências que exigiam que o comportamento persistisse, na ausência de reforço, para só então ele poder ser reforçado. $\mathrm{O}$ contato com tais contingências pode ter estabelecido, não apenas a seqüência CEF, mas também o comportamento de persistir. Essa característica da história desses participantes pode ter interferido na persistência dos comportamentos observados na Fase 3. Assim, do mesmo modo que essa história pode ter contribuído para que os Participantes P32 e P34 tivessem persistido no comportamento de não seguir a regra, mesmo quando este comportamento não produzia ponto, ela também pode ter contribuído para que os Participantes P31 e P33 tivessem persistido no seguimento da regra, mesmo sem ganhar pontos. Além disso, também é possível que estes dois participantes (P31 e P33) tenham seguido a regra, porque o não-seguimento da regra envolvia alto custo de resposta. Isso é, persistir no comportamento de não seguir a regra implicava em claramente desobedecer ao experimentador, que ficava de frente para o participante, embora separado deste pelo espelho unidirecional, monitorando o seguimento da regra (Albuquerque \& cols., 2006).

No Grupo 4 (FR 3/CRF) também ocorreu variabilidade entre os desempenhos dos participantes. Os dados do Participante $\mathrm{P} 42$ podem ser analisados tal como foram analisados os dados dos Participantes P12, P13, P14 e P34, uma vez que ele também emitiu a seqüência CEF imediatamente após deixar de seguir a regra discrepante na Fase 3. O Participante P44 também abandonou seguimento da regra discrepante, mas no caso desse participante não se pode dizer, inequivocamente, que a seqüência ECF ocorreu pela primeira vez na Fase 3, porque a sua ocorrência foi induzida pela história experimental desse participante. Nesse caso, o que se pode dizer é que essa sequiência ocorreu (talvez devido ao contato do seguimento da regra com as consequiências que contradiziam a regra) e foi selecionada por suas consequiências reforçadoras. Os dados do Participante P41, que seguiu a regra em $100 \%$ das tentativas da Fase 3, podem ser analisados tal como foram analisados os dados dos Participantes P31 e P33 do Grupo 3 (FR 3/FR 3). Ou seja, durante a Fase 2, o Participante P41 também pode ter aprendido a persistir e isso pode ter contribuído para que ele tivesse persistido no seguimento da regra. Além disso, na Fase 3, não havia estímulos que pudessem indicar que o não-seguimento da regra seria reforçado em CRF, caso ele ocorresse.

A análise dos resultados do presente estudo sugere que a manutenção do seguimento de regras discrepantes das contingências não depende exclusivamente do esquema programado para reforçar o não-seguimento de regra. Também não depende exclusivamente da história de controle por esquema de reforço. Os dados dos Participantes P11, P12, P13, P14 [Grupo 1 (CRF/CRF)], P32, P34 [Grupo 3 (FR 3/FR 3)] e P42 [Grupo 4 (FR 3/CRF)] sugerem que as conseqüências programadas para o não-seguimento da regra, bem como a história de controle por esquema de reforço de um comportamento alternativo ao especificado pela regra, podem interferir no seguimento de regras discrepantes das contingências. Essa história pode contribuir para determinar a ocorrência do comportamento que substitui o comportamento especificado pela regra discrepante, quando este deixa de ocorrer. Já as consequiências programadas podem contribuir para manter a ocorrência desse comportamento substituto.

Essa análise é consistente com a visão de que uma história experimental exerce controle quando o padrão de respostas apresentado em uma dada fase do experimento continua ou volta a ser apresentado na fase subseqüente (Freeman \& Lattal, 1992; Tatham \& Wanchisen, 1998; Wanchisen, 1990; Weiner, 1969). Já os dados dos Participantes P21, P22, P23 
e P24 [Grupo 2 (CRF/FR 3)], quando comparados com os resultados dos Participantes P11, P12, P13 e P14 [Grupo 1 (CRF/CRF)] sugerem que o contato do não-seguimento de regras com as suas conseqüências reforçadoras é uma condição necessária para que o seguimento de regra deixe de ocorrer definitivamente em uma determinada situação (Albuquerque \& cols., 2004; Paracampo \& Albuquerque, 2004). E os dados dos Participantes P32 e P34 [Grupo 3 (FR 3/FR 3)], quando comparados com os resultados dos Participantes P21, P22, P23 e P24 [Grupo 2 (CRF/FR 3)], sugerem que o seguimento de regras discrepantes das contingências também pode depender, em parte, das exigências das contingências programadas para o comportamento de não seguir a regra ser reforçado e de se o comportamento que satisfaz as contingências programadas para o não-seguimento de regra faz parte ou não do repertório do participante.

Um problema, no entanto, consiste em explicar por que, diferente dos P32, P34 [Grupo 3 (FR 3/FR 3)], P42 e P44 [Grupo 4 (FR 3/CRF)], os Participantes P31, P33 [Grupo 3 (FR 3/FR 3)] e P41 [Grupo 4 (FR 3/CRF)] seguiram a regra discrepante. Não se pode dizer, com segurança, que os Participantes P31, P33 e P41 seguiram a regra porque as contingências programadas eram fracas (Cerutti, 1989; Torgrud \& Holborn, 1990) ou porque eles não discriminaram tais contingências (Baron \& Galizio, 1983; Cerutti, 1989, 1991; Newman \& cols., 1995; Otto \& cols., 1999), uma vez que no presente estudo foi demonstrado controle discriminativo pelas contingências programadas antes da apresentação da regra.

Mas não se pode descartar a possibilidade de que esses participantes tenham seguido a regra discrepante devido a uma história de consequiências mediadas socialmente para o responder de acordo com regras (Hayes, Brownstein, Zettle, Rosenfarb \& Korn, 1986). Por essa proposição, quando uma regra discrepante das contingências é apresentada ao participante, o seu comportamento passaria a ficar sob o controle dessa história de conseqüências mediadas socialmente para o seguimento de regras. O controle por essa história competiria com o controle pelas contingências programadas no experimento e o seguimento da regra seria mantido, ou não, como resultado dessa competição. Assim, poder-se-ia supor que na competição entre o controle exercido por essa história e o controle pelas contingências programadas, prevaleceu o controle pelas contingências programadas no caso dos Participantes P32, P34, P42 e P44, e prevaleceu o controle pela história de consequiências mediadas socialmente no caso dos Participantes P31, P33 e P41. Essa proposição, no entanto, não esclarece quando e sob que condições o controle por essa história pré-experimental seria mais ou seria menos provável de superar o controle pelas conseqüências atuais que seguem o comportamento de seguir regras particulares.

Uma outra possibilidade, então, seria dizer que as diferenças entre os desempenhos desses sete participantes teriam ocorrido devido a diferenças individuais geradas por diferentes histórias pré-experimentais de seguimento de regras (Wulfert \& cols., 1994). Por essa proposição, pessoas que tiveram o comportamento de não seguir regras punido socialmente em uma variedade de situações, ao longo de suas histórias pré-experimentais, tenderiam a desenvolver um repertório de seguir regras inflexível. Já pessoas que tiveram o com- portamento de não seguir regras freqüentemente reforçado socialmente, tenderiam a desenvolver um repertório de seguir regras flexível. Esses autores chegaram a essa conclusão, após observarem que participantes com repertórios classificados como inflexíveis ${ }^{7}$ foram mais prováveis de seguirem a regra discrepante das contingências do esquema múltiplo a que foram expostas do que os participantes classificados de flexíveis. Resultados similares foram encontrados por Pinto, Paracampo e Albuquerque (2006).

Por essa proposição, poder-se-ia supor que os Participantes P32, P34, P42 e P44 teriam uma história pré-experimental em que o comportamento de não seguir regras havia sido freqüentemente reforçado e essa história teria contribuído para que eles tivessem abandonado o seguimento da regra. Já os P31, P33 e P41 teriam uma história pré-experimental em que o comportamento de não seguir regras havia sido freqüentemente punido e essa história teria contribuído para que eles tivessem continuado seguindo a regra. É possível que isso tenha ocorrido, mas as condições experimentais do presente estudo também podem ter contribuído para a ocorrência dessa variabilidade, uma vez que ela ficou restrita ao Grupo 3 (FR 3/FR 3) e ao Grupo 4 (FR 3/CRF).

Tem sido sugerido que o seguimento de regras é provável de ser mantido quando ele é monitorado (Barret, Deitz, Gaydos \& Quinn, 1987; Cerutti, 1994) e é improvável de ser mantido quando ele mantém contato com as consequiências que contradizem a regra (Baron \& Galizio, 1983; Galizio, 1979). Considerando isso, pode-se dizer que os participantes do presente estudo foram expostos a condições que favoreciam tanto a manutenção quanto ao abandono do seguimento da regra. Mas, como já analisado, os participantes do Grupo 1 (CRF/CRF) foram expostos a condições que favoreciam mais ao abandono do que a manutenção do seguimento da regra, e isso pode ter contribuído para que eles tivessem deixado de seguir a regra. Similarmente, os participantes do Grupo 2 (CRF/FR 3) podem ter seguido a regra discrepante possivelmente porque foram expostos a condições que favoreciam mais a manutenção do que ao abandono do seguimento da regra. Já os participantes dos Grupos 3 (FR 3/FR 3) e 4 (FR 3/CRF), foram expostos a condições que favoreciam, de forma mais equilibrada, tanto a manutenção quanto ao abandono do seguimento da regra e a combinação dessas condições pode ter favorecido a variabilidade nos resultados. Essa análise é consistente com a proposição de que a manutenção do seguimento de regras não depende exclusivamente de um ou outro fator particular, mas sim da combinação entre os fatores que favorecem e os que não favorecem o seguimento e o não-seguimento de regras (Albuquerque \& cols., 2003). Por essa proposição, a história pré-experimental seria um dos fatores, e não o único fator, que pode interferir no seguimento de regras. Portanto, os seus eventuais efeitos não seriam exercidos independentemente das variáveis experimentais.

Assim, considerando que a grande parte dos autores, nessa linha de pesquisa, concorda que regras são seguidas

7 Neste estudo, os participantes (estudantes universitários) foram previamente classificados como inflexíveis ou como flexíveis, de acordo com as suas respostas ao questionário desenvolvido por Rehfisch (1958). 
devido a uma história pré-experimental de reforço social para o seguimento e de punição social para o não-seguimento de regras (Baron \& Galizio, 1983; Catania, Shimoff \& Matthews, 1989; Cerutti, 1989; Hayes \& cols., 1986; Joyce \& Chase, 1990; Mallot, 1989; Otto \& cols., 1999; Skinner, 1969; Wulfert \& cols., 1994), pode-se supor que essa história préexperimental contribuiu para que todos os 15 participantes do presente estudo, na transição da Fase 2 para a Fase 3, tivessem deixado de emitir o comportamento que vinha produzindo reforço em suas histórias experimentais e tivessem emitido o comportamento especificado pela regra discrepante. Mas, com base nos resultados do presente estudo, pode-se também supor que as variáveis experimentais no Grupo 1 (CRF/CRF) podem ter impedido a continuação do controle exercido pela história pré-experimental. Já variáveis experimentais no Grupo 2 (CRF/FR 3) podem ter facilitado esse controle. Nos Grupos 3 (FR 3/FR 3) e 4 (FR 3/CRF), algumas variáveis experimentais podem ter contribuído para facilitar, enquanto outras podem ter contribuído para impedir os eventuais efeitos da história préexperimental. Essas suposições são consistentes com a visão de que os efeitos de histórias pré-experimentais podem ser anulados por determinadas variáveis experimentais (Sidman, 1960) e poderiam ser testadas.

Pesquisas futuras, por exemplo, poderiam expor participantes previamente classificados como inflexíveis ou como flexíveis às mesmas condições a que os participantes do presente estudo foram expostos. Assim, se os resultados dos Grupos 1 (CRF/CRF) e 2 (CRF/FR 3) fossem replicados, independentemente da classificação dos participantes e se os resultados dos Grupos 3 (FR 3/FR 3) e 4 (FR 3/CRF) também fossem replicados, mas fosse constatado que os participantes que seguiram a regra haviam sido classificados como inflexíveis e os participantes que abandonaram o seguimento da regra haviam sido classificados como flexíveis, poder-se-ia especificar as condições sob as quais uma história pré-experimental seria mais ou seria menos provável de exercer os seus eventuais efeitos. Por fim, pesquisas futuras também deveriam procurar identificar quais as características que uma história experimental deveria apresentar para exercer a função de substituir ou de manter o comportamento especificado por regras discrepantes. Tais pesquisas também seriam importantes na medida em que pudessem contribuir para identificar outras fontes da variabilidade freqüentemente encontrada nos estudos que têm investigado o seguimento de regras discrepantes em esquemas de reforço intermitente.

\section{Referências}

Albuquerque, L. C. (2001). Definições de regras. Em H. J. Guilhardi, M. B. B. P. Madi, P. P. Queiroz, P. P. \& M. C. Scoz (Orgs.), Sobre comportamento e cognição: Expondo a variabilidade (pp. 132140). Santo André: ARBytes.

Albuquerque, L. C., de Souza, D. G., Matos, M. A. \& Paracampo, C. C. P. (2003). Análise dos efeitos de histórias experimentais sobre o seguimento subseqüiente de regras. Acta Comportamentalia, $11,87-126$.

Albuquerque, L. C., Matos, M. A., de Souza, D. G. \& Paracampo, C. C. P. (2004). Investigação do controle por regras e do controle por histórias de reforço sobre o comportamento humano. Psicologia: Reflexão e Crítica, 17, 395-412.
Albuquerque, L. C., Reis, A. A. \& Paracampo, C. C. P. (2006). Efeitos de uma história de reforço contínuo sobre o seguimento de regra. Acta Comportamentalia, 14, 47-75.

Albuquerque, L. C. \& Silva, F. M. (2006). Efeitos da Exposição a Mudanças nas Contingências Sobre o Seguir Regras. Psicologia: Teoria e Pesquisa, 22, 101-112.

Baron, A. \& Galizio, M. (1983). Instructional control of human operant behavior. The Psychological Record, 33, 495-520.

Barret, D. H., Deitz S. M., Gaydos G. R. \& Quinn P. C. (1987). The effects of programmed contingencies and social conditions on responses stereotipy with human subjects. The Psychological Record, 34, 489-505.

Catania, A. C., Shimoff, E. \& Matthews, A. (1989). An experimental analysis of rule-governed behavior. Em S. C. Hayes (Org.), Rulegoverned behavior: Cognition, contingencies, and instructional control (pp. 119-150). New York: Plenum.

Cerutti, D. T. (1989). Discrimination theory of rule-governed behavior. Journal of the Experimental Analysis of Behavior, 51, 259-276.

Cerutti, D. T. (1991). Discriminative versus reinforcing properties of schedules as determinants of schedule insensitivity in humans. The Psychological Record, 41, 51-67.

Cerutti, D. T. (1994). Compliance with instructions: Effects of randomness in scheduling and monitoring. The Psychological Record, 41, 51-67.

DeGrandpre, R. J. \& Buskist, W. F. (1991). Effects of accuracy of instructions on human behavior: Correspondence with reinforcement contingencies matters. The Psychological Record, 41, 371-384.

Dixon, M. R. \& Hayes, L. J. (1998). Effects of differing instructional histories on the resurgence of rule-following. The Psychological Record, 48, 275-292.

Epstein, R. \& Skinner, B. F. (1980). Resurgence of responding during the cessation of response independent reinforcement. Proceedings of the National Academy of Sciences USA, 77, 6251-6253.

Freeman, T. J. \& Lattal, K. A. (1992). Stimulus control of behavioral history. Journal of the Experimental Analysis of Behavior, 57, $5-15$.

Galízio, M. (1979). Contingency-shaped and rule-governed behavior: Instructional control of human loss avoidance. Journal of Experimental Analysis of Behavior, 31, 53-70.

Hayes, S. C., Brownstein, A. J., Zettle, R. D., Rosenfarb, I. \& Korn, Z. (1986). Rule governed behavior and sensitivity to changing consequences of responding. Journal of the Experimental Analysis of Behavior, 45, 237-257.

Hayes, S. C., Zettle, R. \& Rosenfarb. I. (1989). Rule-following. Em S. C. Hayes (Org.), Rule governed behavior: Cognition, contingencies, and instructional control (pp. 191-220). New York: Plenum.

Joyce, J. H. \& Chase, P. N. (1990). Effects of response variability on the sensivity of rule-governed behavior. Journal of the Experimental Analysis of Behavior, 54, 251-262.

Kaufman, A., Baron, A. \& Koop, R. E (1966). Some effects of instructions on human operant behavior. Psychonomic Monograpg Supplements, 1, 243-250.

LeFrancois, J. R., Chase, P. N. \& Joyce, J. (1988). The effects of variety of instrucions on human fixed-interval performance. Journal of the Experimental Analysis of Behavior, 49, 383-393. 
Lieving, G. A., Hagopian, L. P., Long, E. S. \& O'Connor, J. (2004). Response-class hierarchies and resurgence of severe problem behavior. The Psychological Record, 54, 621-634.

Lieving, G. A. \& Lattal, K. A. (2003). Recency, repeatability, and reinforcer retrenchment: An Experimental Analysis of resurgence. Journal of the Experimental Analysis of Behavior, 80, 217-233.

Malott, R. W. (1989). The achievement of evasive goals: Control by rules describing contingencies that are not direct acting. Em S. C. Hayes (Org.), Rule governed behavior: Cognition, contingencies, and instructional control (pp. 269-322). New York: Plenum.

Martinez, H. \& Tomayo, R. (2005). Interactions of contingencies, instructional accuracy, and instructional history in conditional discrimination. The Psychological Record, 55, 633-646.

Matthews, B. A., Shimoff, E., Catania, A. C. \& Sagvolden, T. (1977). Uninstructed human responding: Sensitivity to ratio and interval contingencies. Journal of the Experimental Analysis of Behavior, 27, 453-467.

Monteles, K. M. C., Paracampo, C. C. P. \& Albuquerque, L. C. (2006). Efeitos de uma história de reforço contínuo e de conseqüências sociais sobre o seguir regras. Psicologia: Reflexão e Crítica, 19, 186-196.

Newman, B., Buffington, D. M. \& Hemmes, N. S. (1995). The effects of schedules of reinforcement on instruction following. The Psychological Record, 45, 463-476.

Otto, T. L., Torgrud, L. J. \& Holborn, S. W. (1999). An operant blocking interpretation of instructed insensitivity to schedule contingencies. The Psychological Record, 49, 663-684.

Paracampo, C. C. P., de Souza, D. G., Matos, M. A. \& Albuquerque, L. C. (2001). Efeitos de mudança em contingências de reforço sobre o comportamento verbal e não verbal. Acta Comportamentalia, 9, 31-55.

Paracampo, C. C. P. \& Albuquerque, L. C. (2004). Análise do papel das conseqüências programadas no seguimento de regras. Interação em Psicologia, 8, 237-245.

Pinto, A. R., Paracampo, C. C. P. \& Albuquerque, L. C. (2006). Análise do controle por regras em participantes classificados de flexíveis e de inflexíveis. Acta Comportamentalia, 14, 171-194.
Rehfisch, J. M. (1958). A Scale for Personality rigidity. Journal of Consulting Psychology, 1, 11-15.

Santos, J. G. W., Paracampo, C. C. P. \& Albuquerque, L. C. (2004). Análise dos efeitos de histórias de variação comportamental sobre o seguimento de regras. Psicologia: Reflexão e Crítica, 17, 413-425.

Shimoff, E., Catania, A. C. \& Matthews, B. A. (1981). Uninstructed human responding: Sensitivity of low-rate performance to schedule contingencies. Journal of the Experimental Analysis of Behavior, 36, 207-220.

Sidman, M. (1960). Tactics of scientific research. Nova York: Basic Books.

Skinner, B. F. (1969). Contingencies of reinforcement: A theoretical analysis. New York: Appleton-Century-Crofts.

Tatham, T. A. \& Wanchisen, B. A. (1998). Behavioral history: A definition and some common findings from two areas of research. The Behavior Analyst, 21, 241-251.

Torgrud, L. J. \& Holborn, S. W. (1990). The effects of verbal performance descriptions on nonverbal operant responding. Journal of the Experimental Analysis of Behavior, 54, 273291.

Wanchisen, B. A. (1990). Forgetting the lessons of history. The Behavior Analyst, 13, 31-37.

Weiner, H. (1969). Controlling human fixed-interval performance. Journal of the Experimental Analysis of Behavior, 12, 349373.

Wulfert, E., Greenway, D. E., Farkas, P., Hayes, S. C. \& Dougher, M. J. (1994). Correlation between self-reported rigidity and rule-governed insensivity to operant contingencies. Journal of Applied Behavior Analysis, 27, 659-671.

Recebido em 01.06.2005

Primeira decisão editorial em 25.09.2006

Versão final em 21.12.2006

Aceito em 28.03.2007

\section{ASSINATURA PARA INSTITUIÇÕES}

Assinaturas de Psicologia: Teoria e Pesquisa podem ser solicitadas diretamente à:

Secretaria de Divulgação

Revista Psicologia: Teoria e Pesquisa

Instituto de Psicologia, Universidade de Brasília

70910-900 Brasília, DF, Brasil.

O valor da assinatura anual é R\$130,00. Número avulso para assinantes custa $R \$ 26,00$ e número antigo $R \$ 20,00$.

Número avulso para não assinantes custa $\mathrm{R} \$ 48,00$. Valores sujeitos a atualização. 